\title{
Doubling the propagation distance of surface plasmon polaritons
}

Victor Pacheco-Peña, Igor V. Minin, Oleg V. Minin, and Miguel Beruete

A novel passive repeater, based on a chain of $3 D$ dielectric particles, has been proposed and numerically simulated.

The performance of modern computing depends directly on the speed of data exchange between cores. Electrical metal interchip connections, however, are fundamentally limited by their bandwidth, ${ }^{1}$ which ultimately determines the maximum data rate. The transition from electronics to photonics-or to nanophotonics, to be precise-may be a possible solution to this problem. ${ }^{2}$ This is because optical computing allows speed and productivity to be increased in proportion to the number of cores. ${ }^{3}$ The fundamental laws of diffraction, however, mean that the size of photonic components cannot be reduced greatly, i.e., their size cannot be smaller than the order of the operation wavelength.

This fundamental problem can be solved by using surface waves instead of volume waves. These surface waves-known as surface plasmon polaritons (SPPs) ${ }^{4}$-are based on the interaction of electromagnetic radiation and conduction electrons on metal surfaces. With the SPP strategy, the diffraction limit of classical optics can be overcome, thanks to the smaller wavelength of the SPPs. The possibility of working in the optical near field, with a sub-wavelength size, also opens up and allows nanoscale devices (about 10nm in size) that operate at high speed (about $10 \mathrm{THz}$ ) to be created. This approach has given rise to an intense amount of research on SPP propagation in the discipline of plasmonics. An important remaining hurdle in the technological development of plasmonics, however, is the relatively short propagation distances of SPPs (caused by their fundamental absorption in metals). ${ }^{4}$ In recent work, photonic nanojets (PNJs) ${ }^{5}$ were proposed at optical wavelengths combined with SPPs, ${ }^{6,7}$ but the propagation distance of the SPPs was small.

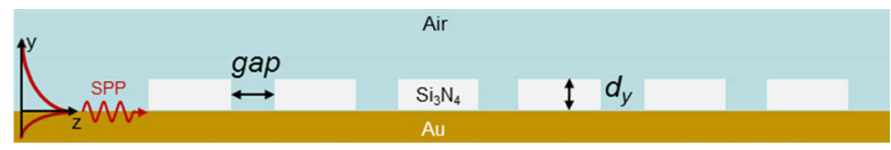

Figure 1. Schematic representation of the surface plasmon polariton (SPP) passive repeater. The structure consists of six separated silicon nitride $\left(\mathrm{Si}_{3} \mathrm{~N}_{4}\right)$ cuboids, with dimensions $d_{x} \times d_{y} \times d_{z}$, placed on top of a thin (100nm) gold (Au) layer.

In the past we have demonstrated that it is possible to employ dielectric cuboids instead of spheres or cylinders ${ }^{5}$ to produce PNJs at sub-terahertz frequencies. We found that this worked for both transmission ${ }^{8,9}$ and reflection ${ }^{10}$ mode regimes. In our most recent work, ${ }^{7}$ we have therefore proposed a new way to increase the propagation distance of surface plasmons at communication wavelengths. Based on our research, we have also reported that a chain of $3 \mathrm{D}$ dielectric cuboids can be used as a waveguide. ${ }^{11}$

In our approach, we can extend the propagation distance of surface plasmons by introducing a chain of cuboids ${ }^{11}$ where SPPs are coupled. ${ }^{7}$ Our proposed structure of passive repeaters is shown schematically in Figure 1. The chain consists of six cuboids that are separated by a gap and placed on top of a thin gold layer. The height of this metal layer is $100 \mathrm{~nm}$, which is less than the skin depth $(30 \mathrm{~nm})$ at a wavelength of $1550 \mathrm{~nm}$. This layer can thus be considered as semi-infinite at the design wavelength. ${ }^{12}$ We use silicon nitride as the low-loss material for the cuboids. This material has a refractive index of 1.97 at the design wavelength. When the whole structure is immersed in air, it has a refractive index of one. As shown in Figure 1, SPPs are excited from the back of the chain and then propagate along the z-axis.

We have also used the commercial CST Microwave Studio ${ }^{\text {TM }}$ software to conduct numerical simulations. In particular, we 


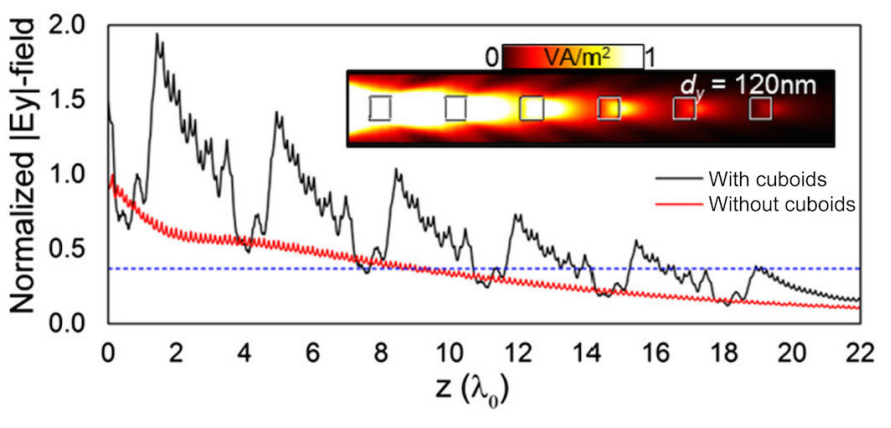

Figure 2. Normalized magnitude of the electric field $\left(E_{y}\right)$ along the propagation axis at the surface of the metal layer in the SPP passive repeater, where $z$ is the propagation distance. These results are for the optimal case, when the air gap between the cuboids is 2.5 times the design wavelength $\left(\lambda_{0}\right)$, the height of the cuboids $\left(d_{y}\right)$ is $120 \mathrm{~nm}$ (black curve), and there is a flat gold film (red curve). The blue curve corresponds to a decay of 1/e, with respect to the amplitude of the incident SPP at the input. The inset shows the normalized power distribution on the metal film for the optimal case. VA/ $\mathrm{m}^{2}$ : Volt-amps per meter squared.

used a dispersive model for gold ${ }^{13}$ in the simulations. Our simulation results ${ }^{7}$ demonstrate that the best propagation distance for an SPP can be obtained when the air gap between the cuboids is 2.5 times the design wavelength and the height of the cuboids is $120 \mathrm{~nm}$. We find it interesting to note that this optimal air gap distance is the same as for the sub-terahertz waveguide. ${ }^{11}$ At this optimal case, the SPP propagation distance is twice the value for the case without cuboids (see Figure 2).

Our simulations also show that the PNJ for each cuboid has sub-wavelength dimensions. Our proposed structure can thus successfully extend the propagation distance of SPPs without deteriorating the focusing characteristics of the PNJs. Through additional investigations we have demonstrated that with the optimal height $(120 \mathrm{~nm})$ of the cuboids, a tolerance of $\pm 40 \mathrm{~nm}$ can be admitted without the propagation distance of the SPPs being strongly changed. This is an important feature for fabrication purposes.

In summary, we have proposed and numerically simulated a novel structure that can be used to significantly increase the propagation distance of surface plasmon polaritons. Our structure - based on a chain of silicon nitride cuboids on top of a thin gold layer-could be applied in the design of several plasmonic devices (e.g., beam steers and SPP optical tweezers). In our future work, we are planning to conduct an experimental demonstration of the SPP repeater.
Author Information

\author{
Victor Pacheco-Peña and Miguel Beruete \\ Public University of Navarra \\ Pamplona, Spain
}

Igor V. Minin, and Oleg V. Minin

National Research Tomsk State University

Tomsk, Russia

\section{References}

1. D. A. B. Miller and H. M. Ozaktas, Limit to the bit-rate capacity of electrical interconnects from the aspect ratio of the system architecture, J. Parallel Distrib. Comp. 41, pp. 42-52, 1997.

2. S. A. Maier, Plasmonics: Fundamentals and Applications, p. 224, Springer, 2007.

3. L. Pavesi and G. Guillot (eds.), Optical Interconnects: The Silicon Approach, p. 384, Springer, 2006.

4. A. V. Zayats, I. I. Smolyaninov, and A. A. Maradudin, Nano-optics of surface plasmon polaritons, Phys. Rep. 408, pp. 131-314, 2005.

5. A. Heifetz, S.-C. Kong, A. V. Sahakian, A. Taflove, and V. Backman, Photonic nanojets, J. Comp. Theoret. Nanosci. 6, pp. 1979-1992, 2009.

6. D. Ju, H. Pei, Y. Jiang, and X. Sun, Controllable and enhanced nanojet effects excited by surface plasmon polariton, Appl. Phys. Lett. 102, p. 171109, 2013. doi:10.1063/1.4802958

7. V. Pacheco-Peña, I. V. Minin, O. V. Minin, and M. Beruete, Increasing surface plasmons propagation via photonic nanojets with periodically spaced $3 D$ dielectric cuboids, Photonics 3, p. 10, 2016. doi:10.3390/photonics3010010

8. V. Pacheco-Peña, M. Beruete, I. V. Minin, and O. V. Minin, Terajets produced by dielectric cuboids, Appl. Phys. Lett. 105, p. 084102, 2014.

9. V. Pacheco-Peña, M. Beruete, I. V. Minin, and O. V. Minin, Multifrequency focusing and wide angular scanning of terajets, Opt. Lett. 40, pp. 245-248, 2015.

10. I. V. Minin, O. V. Minin, V. Pacheco-Peña, and M. Beruete, Localized photonic jets from flat, three-dimensional dielectric cuboids in the reflection mode, Opt. Lett. 40, pp. 2329-2332, 2015.

11. I. V. Minin, O. V. Minin, V. Pacheco-Peña, and M. Beruete, All-dielectric periodic terajet waveguide using an array of coupled cuboids, Appl. Phys. Lett. 106, p. 254102, 2015. doi:10.1063/1.4923186

12. J. J. Burke, G. I. Stegeman, and T. Tamir, Surface-polariton-like waves guided by thin, lossy metal films, Phys. Rev. B 33, pp. 5186-5201, 1986.

13. P. B. Johnson and R. W. Christy, Optical constants of the noble metals, Phys. Rev. B 6, pp. 4370-4379, 1972. 